\title{
Diagnósticos de enfermagem em vítimas de trauma atendidas em um serviço pré-hospitalar avançado móvel
}

\section{Nursing diagnoses in trauma victims attended in a mobile advanced pre-hospital care service}

\author{
Diagnósticos de enfermería en víctimas de trauma atendidas en un servicio \\ pre-hospitalario avanzado mueble
}

\section{Regilene Molina Zacareli CyrilloI, Maria Célia Barcellos Dalri ${ }^{\mathrm{II}}$, Silvia Rita Marin da Silva CaniniII, Emilia Campos de Carvalho ${ }^{\text {IV }}$, Renata Roque Lourenciniv}

\footnotetext{
${ }^{I}$ Enfermeira. Doutoranda do Programa de Enfermagem Fundamental da Escola de Enfermagem de Ribeirão Preto da Universidade de São Paulo (PPGEF/EERP/USP). Ribeirão Preto, SP. E-mail: regizacareli@yahoo.com.br.

II Enfermeira. Doutora em Enfermagem. Docente da EERP/USP. Ribeirão Preto, SP. E-mail: macdalri@eerp.usp.br.

III Enfermeira. Doutora em Enfermagem. Docente da EERP/USP. Ribeirão Preto, SP. E-mail: canini@eerp.usp.br.

IV Enfermeira. Doutora em Enfermagem. Docente da EERP/USP. Ribeirão Preto, SP. E-mail: ecdcava@usp.br.

$\vee$ Enfermeira, Enfermeira da Santa Casa de Misericórdia de Ribeirão Preto, SP. Ribeirão Preto, SP. E-mail: reroque_eerp@yahoo.com.br.
}

\section{RESUMO}

O serviço de atendimento pré-hospitalar móvel de urgência presta atendimento a toda e qualquer solicitação de ajuda fora do âmbito hospitalar visando a manutenção da vida e minimização de sequelas, exigindo do enfermeiro conhecimentos e habilidades específicas. Este estudo teve como objetivo identificar os diagnósticos de enfermagem em vítimas de trauma atendidas em um Serviço Avançado Móvel de Urgência (SAMU) do interior do estado de São Paulo. Foi elaborado e validado um instrumento de coleta de dados, segundo o modelo conceitual de Horta; os dados coletados foram validados por peritos na temática. O projeto foi aprovado por Comitê de Ética e Pesquisa. Foram avaliadas 23 vítimas de trauma, sendo 17 (73,9\%) homens, 16 (69,5\%) com idade entre 18 e 30 anos e 14 (60,1\%) vítimas de acidentes de transito. Os diagnósticos de enfermagem mais frequentes foram: Risco para infecção ( $91 \%$, Risco para trauma (82\%), Dor aguda (74\%), Integridade tissular prejudicada (65\%), Volume de líquidos deficiente $(43 \%)$ e Risco para volume de líquido deficiente (43\%). Este estudo permitiu identificar os principais diagnósticos de enfermagem nesta clientela específica, os quais poderão fornecer importantes subsídios para futuras investigações, sobretudo abordando intervenções de enfermagem.

Descritores: Diagnóstico de Enfermagem; Enfermagem em Emergência; Assistência Pré-Hospitalar.

\section{ABSTRACT}

Mobile pre-hospital emergency care services deliver care to all requests for help outside the hospital environment, aiming at the maintenance of life and minimization of sequelae, demanding specific knowledge and skills from nurses. This study aimed to identify the nursing diagnoses in trauma victims who receive care in a Mobile Advanced Urgency Care Service (SAMU) in the interior of the state of São Paulo, Brazil. A data collection instrument was developed and validated according to Horta's conceptual model. Collected data were validated by experts in the area. The project was approved by the Research and Ethics Committee. Twenty-three trauma victims were evaluated, 17 (74\%) men, 16 (69.5\%) aged between 18 and 30 years old and $14(60.1 \%)$ were victims of traffic accidents. The most frequent nursing diagnoses were: Risk for infection (91\%), Risk for trauma (82\%), Acute pain (74\%), Impaired tissue integrity $(65 \%)$, Deficient fluid volume $(43 \%)$ and Risk for deficient fluid volume $(43 \%)$. The study contributed for the identification of the main nursing diagnoses in this specific clientele. Results can support future researches, mainly addressing nursing interventions.

Descriptors: Nursing Diagnosis; Emergency Nursing; Prehospital Care.

\section{RESUMEN}

El servicio de atención prehospitalaria mueble de urgencia presta atención a toda y cualquiera solicitación de ayuda fuera del ámbito hospitalario, visando a la manutención de la vida y minimización de secuelas, exigiendo del enfermero conocimientos y habilidades específicas. Este estudio tuvo como objetivo identificar los diagnósticos de enfermería en víctimas de trauma asistidas en un Servicio Avanzado Mueble de Urgencia (SAMU) en el interior del estado de São Paulo. Un instrumento de recolecta de datos según el modelo conceptual de Horta fue elaborado y validado. Los datos recolectados fueron validados por expertos en la temática. El proyecto fue aprobado por el Comité de Ética e Investigación. Fueron evaluadas 23 víctimas de trauma, siendo 17 (74\%) hombres, 16 (69,5\%) con edad entre 31 y 40 años, y $14(60,1 \%)$ víctimas de accidentes de tránsito. Los diagnósticos de enfermería más frecuentes fueron: Riesgo para infección (91\%), Riesgo para trauma (82\%), Dolor agudo (74\%), Integridad tisular perjudicada $(65 \%)$, Volumen de líquidos deficiente (43\%) y Riesgo para volumen de líquido deficiente (43\%). Ese estudio permitió identificar los principales diagnósticos de enfermería en esa clientela específica, los cuales podrán apoyar futuras investigaciones, sobre todo relacionadas a las intervenciones de enfermería.

Descriptores: Diagnóstico de Enfermería; Enfermería de Urgencia; Atención Prehospitalaria. 


\section{INTRODUÇÃO}

A partir da década de 80 houve um crescimento na taxa de mortalidade por causas externas, as quais representam a segunda causa de morte no Brasil, sendo os acidentes e os homicídios os maiores responsáveis por este aumento. O trauma é uma lesão de extensão, intensidade e gravidade variável, que pode ser produzida por agentes físicos, químicos, psíquicos e outros, de forma acidental ou intencional, instantânea ou prolongada, em que o poder do agente agressor supera a resistência encontrada(1).

O trauma acarreta consequências sociais e econômicas tanto para os indivíduos quanto para a sociedade. As lesões relacionadas a ele podem ocasionar incapacidades físicas e ou mentais, temporárias ou permanentes e também levar ao óbito(2).

Estudo aponta que o tempo decorrido entre o acidente e $\mathrm{o}$ atendimento hospitalar é um fator decisivo para reduzir a mortalidade e a ocorrência de sequelas, uma vez que $40 \%$ dos óbitos ocorrem na fase pré-hospitalar ${ }^{(2)}$; em face destas características, para $o$ atendimento neste cenário, há a necessidade de profissionais capacitados.

Destaca-se que, no Brasil, a implantação dos serviços de atendimento pré-hospitalar ocorreu no início da década de 90, o qual passou a ser denominado de Serviço de Atenção Móvel de Urgência (SAMU), a partir do Plano Nacional de Atendimento à Urgência e Emergência em 2003(3).

Neste novo contexto, a área de urgência e emergência tem exigido dos enfermeiros uma nova forma de atuação, tornando-se necessário o rompimento com um modelo de trabalho tradicional cujas tarefas, em sua maioria, são baseadas no modelo de assistência biomédico e centradas em rotinas e tarefas, o qual não tem dado solução às necessidades humanas do paciente crítico.

O Conselho Federal de Enfermagem (COFEN) estabeleceu, pela lei no. 7498 de 1986, a implementação da sistematização da assistência de enfermagem nas instituições de saúde, cuja responsabilidade privativa é do enfermeiro(4). Segundo a legislação(5), que dispõe sobre a regulamentação da Assistência de Enfermagem em Atendimento Pré-Hospitalar e demais situações relacionadas com o Suporte Básico e Suporte Avançado de Vida, somente o enfermeiro poderá realizar procedimentos de alta complexidade e prestar assistência de enfermagem em unidades móveis de UTI e Suporte Avançado de Vida terrestre, aéreo ou aquático.

Ainda, é privativo do enfermeiro o estabelecimento de diagnósticos de enfermagem; sua elaboração permite a composição de uma ampla rede documental de informações codificadas, de conceitos e evidências científicas, que certamente contribuem para o delineamento de conhecimentos da profissão, sobretudo na escolha de intervenções e resultados esperados.

Diante deste panorama e considerando a escassez de estudos abordando os diagnósticos de enfermagem nesta clientela específica, julgou-se oportuna a realização da presente investigação que teve como objetivo identificar os diagnósticos de enfermagem presentes em vítimas de trauma, atendidas num Serviço de Atenção Móvel de Urgência (SAMU) do interior paulista.

\section{METODOLOGIA}

Trata-se de um estudo descritivo de caráter prospectivo, realizado em um Serviço de Atenção Móvel de Urgência (SAMU), da Secretaria Municipal de Ribeirão Preto, após ter recebido anuência da referida Secretaria e ter sido aprovado pelo Comitê de Ética em Pesquisa da Escola de Enfermagem de Ribeirão Preto da Universidade de São Paulo (Protocolo no 0411/2003), devidamente reconhecido pela Comissão Nacional de Ética em Pesquisa (CONEP) do Conselho Nacional de Saúde (CNS).

Para a coleta de dados foi elaborado um instrumento fundamentado no modelo Conceitual de

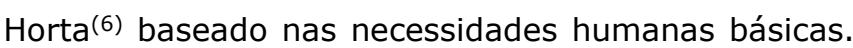
Este instrumento foi submetido à apreciação de cinco especialistas, aos quais foi solicitado avaliar a forma de apresentação, facilidade de compreensão e adequação para o alcance dos objetivos do estudo, ou seja, a validação da forma e conteúdo. Depois de acatadas as sugestões dos especialistas, considerouse 0 instrumento ${ }^{(7)}$ pertinente para 0 alcance dos objetivos, procedeu-se então a sua a aplicação, por uma das pesquisadoras, a cinco vítimas de trauma. Não houve nesta etapa dificuldades de preenchimento do instrumento e estas coletas não foram incluídas no presente estudo.

Os dados foram coletados no período de janeiro a julho de 2004, por uma das pesquisadoras, durante o atendimento pré-hospitalar da vítima de trauma. A coleta encerrava-se no momento que o cliente era transferido para a unidade de tratamento definitivo. $O$ registro dos dados no instrumento de pesquisa era realizado após o atendimento inicial. Os 23 pacientes adultos que compuseram este estudo foram selecionados por meio de amostra de conveniência. Foram incluídos no estudo, sujeitos maiores de 18 anos e que concordaram em participar do estudo mediante a assinatura do Termo de Consentimento Livre e Esclarecido, sendo que nos casos em que o paciente estava impossibilitado essa solicitação foi feita à família ou ao responsável. O consentimento foi solicitado às vitimas de trauma, após sua estabilização e nos casos em que estavam impossibilitadas aguardava-se a chegada da família no local para onde 0 paciente havia sido 
encaminhado. Um dos pesquisadores fazia parte da equipe do Serviço de Atenção Móvel de Urgência e foi responsável por coletar todos os dados.

Após a coleta de dados procedeu-se à análise e síntese dos dados, seguindo o modelo proposto por Risner ${ }^{(8)}$, e ao estabelecimento dos diagnósticos de enfermagem, segundo a Taxonomia II da North American Nursing Diagnosis Association(9).

Os instrumentos de coleta de dados preenchidos, os registros do raciocínio diagnóstico e os diagnósticos de enfermagem identificados foram entregues a cinco enfermeiros peritos em trauma e em diagnósticos de enfermagem. Em seguida, foi solicitada aos mesmos que confirmassem ou não a presença dos diagnósticos. A freqüência de concordância entre os enfermeiros peritos $e$ a pesquisadora foi de $98 \%$.

\section{RESULTADOS E DISCUSSÃO}

Observa-se que a maioria dos sujeitos era do gênero masculino 17 (73,9\%), 16 (69,6\%) tinham idade entre 18 e 30 anos; o tipo de ocorrência mais frequente foi acidente relacionado ao trânsito como atropelamento, acidente automobilístico, motociclístico e ciclístico, que juntos representaram $14(60,8 \%)$ das ocorrências, seguido por ferimento por arma de fogo em $04(17,4 \%)$ vítimas e arma branca $02(8,7 \%)$ vítimas (Tabela 1).

Tabela 1: Caracterização das vítimas de trauma avaliadas no APH Avançado Móvel, Ribeirão Preto, 2004.

\begin{tabular}{|c|c|c|}
\hline Variáveis & No & $\%$ \\
\hline \multicolumn{3}{|l|}{ Sexo } \\
\hline Masculino & 17 & 73,9 \\
\hline Feminino & 06 & 26,1 \\
\hline \multicolumn{3}{|l|}{ Idade (anos) } \\
\hline $18-30$ & 16 & 69,6 \\
\hline $31-40$ & 04 & 17,4 \\
\hline $41-50$ & 02 & 8,7 \\
\hline$\geq 51$ & 01 & 4,3 \\
\hline \multicolumn{3}{|l|}{ Tipo de ocorrência } \\
\hline Atropelamento & 04 & 17,4 \\
\hline Acidente Automobilístico & 04 & 17,4 \\
\hline Acidente Motociclístico & 04 & 17,4 \\
\hline Ferimento por Arma de Fogo & 04 & 17,4 \\
\hline Ferimento por Arma Branca & 02 & 8,7 \\
\hline Acidente Ciclístico & 02 & 8,7 \\
\hline Ferimento Transfixante & 01 & 4,3 \\
\hline Espancamento & 01 & 4,3 \\
\hline Queda & 01 & 4,3 \\
\hline
\end{tabular}

As características socioeconômicas, políticas e culturais de cada região exercem papel importante na causa de ocorrências de acidentes. Estudo apontou que homens adultos jovens são os mais acometidos por agravos traumáticos, com predominância de acidentes de trânsito ${ }^{(1,10)}$.

Na última década, no Brasil, os acidentes de trânsito cresceram substancialmente, em decorrência do aumento do número de veículos em circulação, da desorganização do trânsito, da deficiência geral de fiscalização das condições dos veículos e do comportamento dos usuários, aliados à impunidade dos infratores ${ }^{(1)}$.

$\mathrm{Na}$ tabela 2 encontra-se a distribuição dos diagnósticos de enfermagem propostos pela Taxonomia II da $\operatorname{NANDA}^{(9,11)}$, a partir do modelo conceitual das Necessidades Humanas Básicas de Horta(6), segundo cada uma das subcategorias de necessidades. Observa-se que as necessidades de integridade física, circulação e percepção sensorial foram as mais frequentes. 
Tabela 2: Distribuição dos diagnósticos de enfermagem ( $N=176)$ identificados nas vítimas de trauma atendidas no APH Avançado Móvel, segundo as Categorias e Subcategorias

Humanas Básicas de Horta, Ribeirão Preto, 2004.

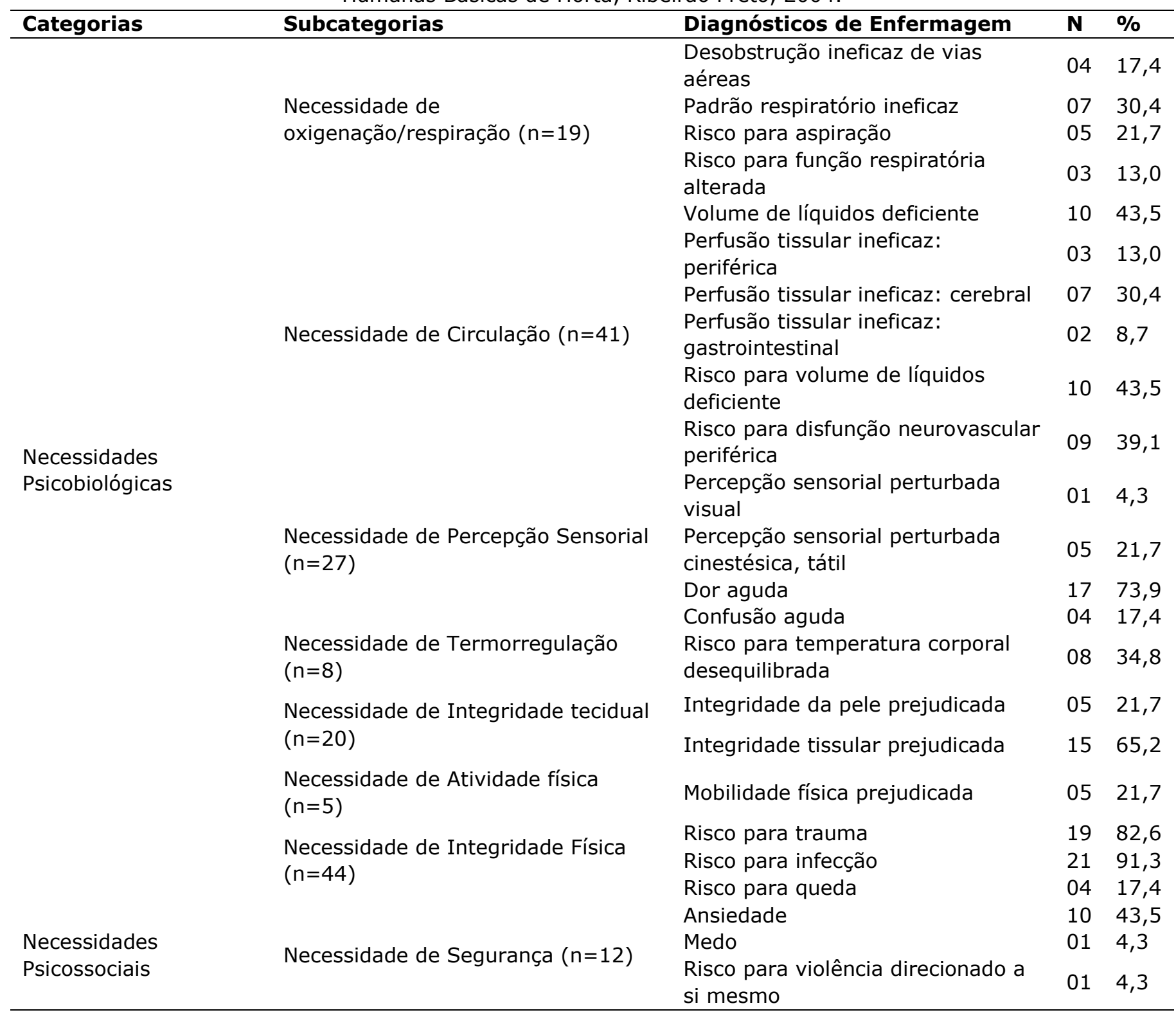

Em relação à avaliação das vítimas, utilizou-se o modelo conceitual de Horta(6) o qual está centrado no atendimento das necessidades humanas básicas, classificadas segundo o nível de hierarquia em Psicobiológicas, Psicossocial e Psicoespiritual.

$\mathrm{Na}$ categoria Psicoespiritual não foi identificada necessidade alterada, nas condições do presente estudo. Já na categoria de Necessidades Psicobiológicas foram identificadas sete subcategorias: oxigenação/respiração, circulação, percepção sensorial, termorregulação, integridade tecidual, atividade física e integridade física; e uma nas Necessidades Psicossociais: necessidade de segurança, sendo que a satisfação de todas as subcategorias é indispensável à necessidade humana básica de sobrevivência física.

Dentre a categoria de Necessidades Psicobiológicas foram identificados 21 (87,5\%) diagnósticos de enfermagem e na categoria das Necessidades Psicossociais, três (12,5\%), estabelecidos para as 23 vítimas de trauma atendidas no APH Avançado Móvel.

$\mathrm{Na}$ subcategoria de Necessidade de oxigenação/respiração foram identificados os seguintes diagnósticos de enfermagem: Desobstrução ineficaz de vias aéreas em quatro vitimas $(17,4 \%)$; Padrão respiratório ineficaz em sete $(30,4 \%)$; Risco para aspiração em cinco $(21,7 \%)$ e Risco para função respiratória alterada em três $(13 \%)$ delas. A necessidade de o organismo obter oxigênio por meio da ventilação, da difusão e perfusão e da eliminação de dióxido de carbono é definida como necessidade de oxigenação, sendo imperativa para a sobrevivência das pessoas e assim produzir energia para manter a vida(12). As necessidades fisiológicas devem ser atendidas pelo 
menos em um nível mínimo, para a sobrevida do corpo ou da espécie. Assim, nas situações de emergência, as necessidades fisiológicas têm prioridade em relação às demais, mas independente disto o enfermeiro deverá estar atento a interação de todas as outras necessidades ${ }^{(7)}$.

Os diagnósticos de enfermagem pertencentes a esta subcategoria determinam um grupo de evidências clínicas e de respostas humanas que podem aumentar a vulnerabilidade das vítimas de trauma, devendo então ser identificados por meio de um rápido exame clínico, com freqüência compilada em função da gravidade e do número de vítimas existentes na cena do acidente. Além disto, os diagnósticos devem ser precisos e estabelecidos com rapidez, sendo que implementação das intervenções essenciais deve ser instituída simultaneamente à coleta de dados.

Para a Necessidade de Circulação foram identificados seis diagnósticos de enfermagem: Volume de líquidos deficiente em 10 (43,5\%) vítimas; Perfusão tissular ineficaz: periférica em três $(13,0 \%)$ vítimas; Perfusão tissular ineficaz: cerebral em sete $(30,4 \%)$ vítimas; em duas $(8,7 \%)$ vítimas Perfusão tissular ineficaz: gastrointestinal; Risco para volume de líquidos deficiente em 10 (43,5\%) e Risco para disfunção neurovascular periférica em nove $(39,1 \%)$ vítimas. Esta necessidade é imprescindível para transportar e distribuir nutrientes vitais ao organismo e remover substâncias desnecessárias. É responsável por manter os líquidos corporais em níveis adequados, e assim favorecer o metabolismo corporal, possibilitando a homeostase e a sobrevivência do organismo(1,13).

O conhecimento do enfermeiro sobre as necessidades de oxigenação/respiração e circulação é fundamental para a detecção precoce das alterações que ocorrem com as vítimas atendidas pelo $\mathrm{APH}$ Móvel cuja finalidade é prevenir, principalmente, o agravamento da situação na cena do acidente.

Para a identificação das respostas do indivíduo ao trauma o enfermeiro deve possuir uma base sólida em semiologia, anatomia e fisiopatologia, agregando sua experiência clínica e de suas habilidades de interação e observação. Entende-se assim, que esse conjunto de habilidades é essencial para o julgamento clínico e consequente tomada de decisão. Os cuidados pré-hospitalares podem fazer a diferença entre a vida e a morte, entre uma sequela temporária, grave, ou permanente, ou ainda, entre uma vida produtiva e uma destituída de bem estar. As intervenções que auxiliam o enfermeiro neste cenário do APH estão embasadas em diretrizes preconizadas universalmente(13).

O ser humano necessita preservar e/ou restabelecer o funcionamento do sistema nervoso com o objetivo de controlar e coordenar as atividades do corpo e alguns aspectos do comportamento, por meio da percepção e interação com o meio ambiente resultando na necessidade de percepção sensorial(11,14). Nesta necessidade foram identificados os seguintes diagnósticos de enfermagem: Percepção sensorial perturbada visual em uma $(4,3 \%)$ vítima; Percepção sensorial perturbada cinestésica, tátil em cinco $(21,7 \%)$; Dor aguda em $17(73,9 \%)$ e Confusão aguda em quatro $(17,4 \%)$ delas.

Destaca-se que a dor é uma das principais conseqüências do trauma(15) e a dor aguda foi um diagnóstico frequente no presente estudo. Apesar de ser subjetiva, a dor é considerada uma experiência sensorial e emocional desagradável, e cada indivíduo aprende o seu significado, mediante experiências relacionadas com eventos vividos. No caso da vítima de trauma, geralmente a dor está associada à lesão tecidual, presente ou em potencial e pode causar diversos prejuízos ao organismo. Estudo(16) realizado com a equipe de um serviço de emergência identificou que tanto médicos como enfermeiros tem dificuldades em conceituar dor e ainda apresentam divergências em relação a indicação ou não da terapêutica analgésica. Os autores consideraram fundamental a valorização da dor no paciente vítima de trauma e concluíram que a capacitação dos profissionais para esta avaliação pode contribuir para a qualidade.

Vários fatores podem dificultar o relato de dor pelo paciente como a alteração do nível de consciência, a dificuldade de comunicação relacionada aos dispositivos orotraqueais e endotraqueais, bem como a confusão mental. Daí a importância da equipe de saúde identificar também respostas autonômicas como hipotensão, aumento da freqüência de pulso, sudorese e extremidades frias, as quais podem ser indicativas deste diagnóstico. O enfermeiro do APH Avançado Móvel deve conhecer os fatores que geram as forças da mecânica do trauma, pois podem influenciar a intensidade da dor na vítima (13).

A subcategoria "necessidade de termorregulação" é explicada pela necessidade do organismo manter a temperatura corporal entre $36^{\circ} \mathrm{C}$ e $37,70 \mathrm{C}$, isto ocorre por meio da produção e perda de energia térmica, regulada por mecanismos nervosos de realimentação, e operam por meio de centros termorreguladores do hipotálamo. O organismo, para manter as condições ideais necessita ter sua temperatura corporal dentro de limites muito estreitos, logo temperaturas acima ou abaixo deste limite podem comprometer outras necessidades(13). No presente estudo identificou-se Risco para temperatura corporal desequilibrada em oito $(34,8 \%)$ vítimas de trauma, pesquisa(17) apontou que o trauma foi a segunda condição relacionada aos casos 
de hipotermia em pacientes admitidos num hospital terciário de Israel.

No trauma, a vítima pode perder calor por convecção, quando exposta ao movimento de corrente de ar que resfria o corpo, resultando em uma perda de até $12 \%$ do calor corporal total; ou por condução decorrente, contato direto com objetos como, por exemplo, a administração de soluções em temperaturas menores que a corporal. A hipotermia, no ser humano, interfere em diversas funções fisiológicas como metabolismo basal, transporte de oxigênio e gás carbônico, concentrações de íons hidrogênio no sangue, nos sistemas nervoso, cardiovascular, respiratório, urinário, digestivo e nas alterações hematológicas, hídricas, eletrolíticas e hormonais $^{(1,13)}$.

Para a subcategoria "necessidade de integridade tecidual" foram identificados os diagnósticos de enfermagem: Integridade da pele prejudicada em 05 $(21,7 \%)$ vítimas e Integridade tissular prejudicada, em $15(65,2 \%)$. A pele constitui-se na barreira primária de proteção, sendo de fundamental importância que as características de sensibilidade, elasticidade, vascularização, umidade e coloração do tecido epitelial, subcutâneo e mucoso sejam preservadas. É considerado o maior órgão do corpo humano, portanto está mais sujeita a sofrer ferimentos do que qualquer outro tecido, pois reveste toda superfície externa do organismo(13).

Durante $\mathrm{o}$ atendimento às vítimas trauma, o enfermeiro do APH Avançado Móvel, deve avaliar a perda da integridade de tecidos conectivos, músculos, tendões e tecido conjuntivo ósseo os quais constituem uma ameaça pelo risco de sangramento, infecção e trauma secundário, apontados como fatores determinantes de sobrevida(13).

Em todos os casos os mecanismos do trauma determinam o contato de uma superfície com os tecidos, transferindo energia cinética ou por pressão. Conforme a dimensão e a forma da superfície que transfere a energia ao corpo da vítima podem classificar os agentes mecânicos, e as lesões por eles produzidas em contundentes, perfurantes, cortantes, perfuro-contundentes, perfurocortantes, cortocontundentes. Esses agentes geram respectivamente, lesões contusas, puntiformes, incisas, perfuro contusas, perfuro incisas e corto $\operatorname{contusas}^{(1,13,18)}$.

Assim, na presença do diagnóstico de enfermagem Integridade Tissular Prejudicada, o foco de atenção do enfermeiro no APH Avançado Móvel será a integridade estrutural e função fisiológica da pele. Portanto, a avaliação das lesões deve levar em conta o risco de infecção, o controle da temperatura, o controle de sangramento; uma vez que podem comprometer também outras categorias diagnósticas alocadas nas necessidades de circulação, sensopercepção e termorregulação. O conhecimento sobre o mecanismo do trauma pode prover dados às equipes do $\mathrm{APH}$ e do atendimento hospitalar e consequentemente propiciar a identificação precoce de possíveis danos complexos na área da lesão ${ }^{(13,18)}$.

Já a necessidade de integridade física é liderada pela mecânica corporal que inclui o conhecimento da atividade de certos grupos musculares que são utilizados para, seguramente gerar e manter o movimento. A regulação do movimento engloba o funcionamento integrado dos sistemas ósseos, músculoesquelético e nervoso. A mobilidade serve para expressar um gesto não-verbal, autodefesa, satisfação de outras necessidades e, para seu funcionamento adequado, é preciso que os sistemas nervoso, muscular e ósseo estejam íntegros e funcionando(18). Nesta subcategoria identificou-se o diagnóstico de Mobilidade física prejudicada em cinco indivíduos $(21,7 \%)$.

O diagnóstico de enfermagem Mobilidade física prejudicada é definido pela NANDA(11) como "limitação no movimento físico independente e voluntário do corpo ou de uma ou mais extremidades". As lesões decorrentes de eventos traumáticos que acometem essa necessidade resultam em deficiências e incapacidades temporárias ou permanentes as quais podem comprometer a capacidade para as atividades diárias, bem como, a qualidade de vida(10). Este diagnóstico foi identificado nas vítimas acometidas por arma de fogo, ferimento transfixante, acidente motociclístico e atropelamentos.

Nessas situações, o tipo de lesão mais frequente são as fraturas, as contusões e as luxações, que acarretam longos períodos de recuperação da vítima com importantes custos econômicos e sociais ${ }^{(10,18)}$.

A manutenção da necessidade da integridade física compreende a redução ou eliminação das ameaças biológicas, do corpo; nesta subcategoria foram identificados os seguintes diagnósticos de enfermagem: Risco para trauma em 19 (82,6\%) vítimas; Risco para infecção em 21 (91,3\%) e Risco para queda em quatro $(17,4 \%)$.

No atendimento pré-hospitalar a ameaça pode ser a doença preexistente, o tipo de trauma ocorrido, a falta de segurança no local da cena do acidente ou a própria exposição ambiental, portanto a segurança deve ser prevista tanto para a equipe do $\mathrm{APH}$, como para o paciente. Nesses casos, a equipe do APH deverá remover o paciente, imediatamente para um ambiente seguro antes de iniciar a avaliação e as intervenções necessárias ${ }^{(13)}$; devendo esta necessidade, muitas vezes, ter prioridade à necessidade biológica.

O diagnóstico de enfermagem Risco para Infecção é conceituado pela NANDA"(11) como: "estar em risco aumentado de ser invadido por organismos patogênicos"; ele foi estabelecido em 21(91\%) 
vítimas. No cenário do APH Móvel Avançado reduzir a infecção por microorganismos patogênicos é fundamental. As principais causas de mortalidade das vítimas de traumas físicos, no hospital, são o choque hipovolêmico, nas primeiras horas, e a infecção é a causa tardia mais frequente(18-19).

A infecção é uma das mais frequentes e graves complicações das vítimas de trauma, principalmente, na fase dos cuidados em Unidades de Terapia Intensiva; ela é considerada um dos maiores desafios para a equipe de saúde, após a fase da hora de "ouro", na qual a avaliação inicial e secundária da vítima são prioridades máximas. Tanto as alterações imunológicas relacionadas ao próprio trauma como os inúmeros procedimentos invasivos aumentam a predisposição desta clientela à infecção(1,13,18).

A alta mortalidade dos pacientes vítimas de trauma, que evoluem para sepse, choque séptico ou inúmeras disfunções orgânicas, sustenta a preocupação que o profissional do APH deverá ter para evitar qualquer risco adicional de infecção em uma vítima de trauma, uma vez que elas podem apresentar perda da integridade da pele(1,13,18). Destaca-se que muitas vezes, as condições da vítima e do próprio local do acidente não permitem a adoção de medidas de assepsia, principalmente na instalação de dispositivos invasivos, cabendo a equipe do $\mathrm{APH}$ comunicar este fato à equipe de atendimento hospitalar, a qual deve providenciar a troca dos dispositivos tão logo a vítima esteja estabilizada.

A pele íntegra constitui uma barreira mecânica contra a invasão de microorganismos, além de secretar vários agentes antimicrobianos, porém, no trauma físico é a primeira barreira a ser rompida(19). A contaminação da ferida traumática é inevitável e a inoculação das bactérias endógenas e exógenas é facilitada pela rotura dos epitélios de defesa, pela penetração de corpos estranhos, pelo acúmulo de transudado nas áreas lesadas. Ocorre uma profunda desorganização dos mecanismos imunológicos de defesa após traumatismos físicos, facilitando a agressão por parte das bactérias e inicia-se uma verdadeira síndrome de imunodeficiência adquirida pós-traumática, de efeitos devastadores $(1,13,19)$.

A higienização das mãos dos profissionais que atendem as vítimas deve ser enfatizada, assim como o cuidado com a limpeza diária da viatura e a limpeza e desinfecção dos aparelhos e equipamentos utilizados na assistência à vítima; o respeito ao conceito de assepsia e esterilidade na execução dos procedimentos invasivos e o uso de equipamentos individuais de proteção são meios de oferecer um cuidado qualificado no âmbito do APH Avançado Móvel com relação aos fatores de risco para infecção.

A NANDA ${ }^{(11)}$ define o diagnóstico de Risco para trauma como "risco acentuado de lesão tecidual acidental (p.ex., ferida, queimadura, fratura)". Esse diagnóstico de enfermagem foi estabelecido em do presente estudo. O diagnóstico de enfermagem Risco de trauma foi identificado em 19 (82\%) vítimas de trauma, exceto nas acometidas, por ferimento de armas de fogo e branca, em membros inferiores e superiores.

Alguns fenômenos que ocorrem na cinética da energia, como, a desaceleração, aceleração são fatores predisponentes para alguns tipos de lesões nas vítimas de traumas. Esses fatores de risco são bases de conhecimento sobre essa temática, na busca de evidências para o diagnóstico, na cena do acidente $^{(13)}$. Portanto, o enfermeiro deve investigar o risco potencial de lesão de cada vítima; associando as avaliações da necessidade de sensopercepção com o contexto ambiental.

Nem todos os riscos ambientais presentes na cena do acidente podem ser controlados, entretanto o reconhecimento do mecanismo de trauma pode contribuir para reduzir a possibilidade de outras lesões. O manejo incorreto de uma vítima de trauma é considerado uma imperícia e pode ocasionar uma iatrogênia grave como, por exemplo, o manuseio incorreto de uma vítima com fratura de coluna cervical que poderá resultar em secção da medula e causar um dano irreversível. Esse fator de risco deve ser considerado também nas vítimas de ferimento por arma de fogo e arma branca na região do pescoço e tórax, posto que, por muitas vezes o projétil pode lesar a coluna em algum de seus segmentos $^{(13)}$.

A prevenção de lesões exige, da equipe, a aplicação de estratégias passivas e ativas capazes de proporcionar proteção automática durante o atendimento, o uso do colar cervical, protetor lateral de cabeça, prancha rígida, dentre outros equipamentos e materiais que reduzem os riscos de trauma e lesão(13).

$\mathrm{Na}$ categoria de necessidade psicossocial identificou-se a subcategoria de necessidade de segurança contemplando os seguintes diagnósticos: Ansiedade em 10(43,5\%) vítimas; Medo em uma $(4,3 \%)$ e Risco para violência direcionado a si mesmo em uma $(4,3 \%)$.

Segundo a NANDA(11) o diagnóstico de Ansiedade é definido como "um vago e incômodo sentimento de desconforto ou temor, acompanhado por uma resposta autonômica (a fonte é frequentemente nãoespecífica ou desconhecida para o indivíduo); um sentimento de apreensão causado pela antecipação de perigo. É um sinal de alerta que chama a atenção para um perigo iminente ao indivíduo tomar medidas para lidar com a ameaça". Este diagnóstico foi identificado em 10 (43,5\%) vítimas de trauma.

Ansiedade e medo são diagnósticos de enfermagem frequentemente estabelecidos na prática clínica e discutidos na literatura de enfermagem. 
Esses conceitos são objetos de pesquisas

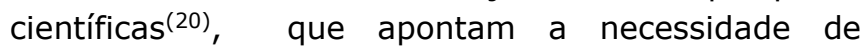
investigações em diferentes contextos e eventos clínicos.

No presente estudo não foram identificados diagnósticos na dimensão psicoespiritual, o que pode ser atribuído ao tipo de atendimento ou mesmo ao despreparo da equipe para a obtenção de dados relacionados a esta dimensão; merecendo assim, ser foco de futuras investigações.

\section{CONCLUSÃO}

O presente estudo evidenciou um predomínio de diagnósticos de enfermagem na categoria de Necessidades Psicobiológicas, necessidades que não atendidas prontamente, representa grave ameaça à vida. Porém, ao estabelecer prioridades, o enfermeiro do APH Móvel Avançado, não pode desconsiderar que as necessidades humanas básicas são interrelacionadas; deve também estar atento aos demais sistemas corporais, ambiente, valores, ética e cultura. Portanto, a dinâmica da assistência de enfermagem, a organização do atendimento, a identificação dos problemas reais e de risco, com base no julgamento clínico e tomada de decisão devem ser o principal foco de atenção do enfermeiro e da equipe do APH Móvel Avançado.

A sistematização de enfermagem na prática clínica compõe o campo de ação da enfermagem científica, baseada nas evidências e na construção de um saber legítimo da profissão. Assim, identificar diagnósticos de enfermagem das vítimas de trauma, no APH avançado Móvel, possibilita aos enfermeiros que atuam neste cenário detectar e controlar os riscos precocemente e planejar individualmente o cuidado prestado a esses pacientes por meio de intervenções específicas, fundamentadas cientificamente, capazes de proporcionar ações eficazes e imediatas.

Diante da escassez de estudos sobre a sistematização da assistência a vitimas de trauma, este estudo permitiu identificar os principais diagnósticos de enfermagem nesta clientela específica, os quais poderão fornecer importantes subsídios para futuras investigações, sobretudo abordando intervenções de enfermagem.

\section{REFERÊNCIAS}

1. Freire E. Trauma: a doença dos séculos. São Paulo: Ed. Atheneu; 2001.

2. Mock CN, Jurkovich GJ, Amon-Kotei D, ArreolaRisa C, Maier RV. Trauma mortality patterns in three nations at different economic levels: implications for global trauma system development. J Trauma 1998;44(5):804-12.

3. Portaria no. 1864 de 29 de setembro de 2003. Institui o componente pré-hospitalar móvel da
Política Nacional de Atenção às Urgências. Diário Oficial da União (Brasília). 2003 Out 5.

4. Portal COFEN [Internet]. Brasília: Conselho Federal de Enfermagem (BR) [update 2008 jun 16, cited 2008 jul 24]. Resolução COFEN-272/2002. Dispõe sobre a sistematização da Assistência de Enfermagem - SAE - nas Instituições de Saúde Brasileiras. Available from: http://corensp.org.br/072005/legislacoes/legislacoes busca.php?leg id=10086\&texto.

5. Conselho Regional de Enfermagem - SP. Decisão COREN-SP/DIR/01/2001. Dispõe sobre a regulamentação de assistência de enfermagem em atendimento pré-hospitalar e demais situações relacionadas ao suporte básico e avançado de vida. São Paulo: Conselho Regional de Enfermagem; 2001. 6. Horta WA. Processo de Enfermagem. São Paulo: EPU; 1979.

7. Cyrillo RMZ, Dalri MCB, Cristina JÁ. Construção e validação do instrumento de coleta de dados para a assistência de enfermagem no atendimento préhospitalar móvel avançado a vítimas de trauma. Rev. Rene. 2005;6(2):55-60.

8. Risner PB. Diagnosis: analysis and synthesis of data. In: Christensen PJ; Kenney JW. Nursing process: conceptual models. St. Louis: Mosby; 1990. p. $158-78$.

9. NANDA - North American Nursing Diagnosis Association. Diagnósticos de enfermagem: definições e classificações. Porto Alegre: Artes Médicas; 2002.

10. Malvestio MA, Sousa RMC. Acidentes de trânsito: caracterização das vítimas segundo o "Revised Trauma Score" medido no período pré-hospitalar. Rev. esc. enferm. USP. 2002;36(4):394-401.

11. NANDA - North American Nursing Diagnosis Association. Diagnósticos de enfermagem: definições e classificações. Porto Alegre: Artes Médicas; 2008.

12. Dalri MCB, Rossi LA, Cyrillo RMZ, Canini SRMS, Carvalho EC. Validação do diagnóstico troca de gases prejudicada em adultos no atendimento de emergência. Cien enfem 2008;14(1):63-72.

13. PHTLS basic and advanced Prehospital Trauma Life Support. 6 nd. St Louis: Mosby; 2007.

14. Feitoza DS, Freitas MC, Silveira RE. Traumatismo crânioencefálico: diagnósticos de enfermagem a vítimas atendidas em UTI. Rev. Eletr. Enf. [Internet]. 2004 [cited 2009 dez 30];6(2):223-33. Available from:

http://www.fen.ufg.br/revista/revista6 2/pdf/Orig9 t ce.pdf.

15. McCaffery, Margo; Pasero, Chris. Am J Nurs. 2001;101(11):13.

16. Calil AM, Pimenta CAM. Conceitos de enfermeiros e médicos de um serviço de emergência sobre dor e analgesia no trauma. Rev. esc. enferm. USP. 2009;39(3):325-32. 
17. Elbaz G, Etzion O, Delgado J, Porath A, Talmor D, NovackV. Hypothermia in a desert climate: severity score and mortality prediction. Am j Emerg Med. 2008;26(6):693-8.

18. Birolini D, Utiyama, E, Steinman E. Cirurgia de emergência com teste de auto- avaliação São Paulo: Atheneu; 2001.

19. Fernandes AT, Fernandes MOV, Ribeiro Filho N. Infecção hospitalar e suas interfaces na área da saúde. São Paulo: Atheneu; 2000.

20. Lehto RH, Stein KF. Death anxiety: na analysis of na evolving concept. Res Theory Nur Pract. 2009;23(1):23-41.

Artigo recebido em 14.10.08.

Aprovado em 08.09.09.

Publicado em 31.12.09. 